\title{
The Generalized Solutions of a System of Maxwell's Equations for the Uniaxial Anisotropic Media
}

\author{
Seil Sautbekov \\ Eurasian National University \\ Kazakhstan
}

\section{Introduction}

Media with anisotropic properties are widely used in modern radio electronics, an astrophysics, and in plasma physics. Anisotropic materials have found wide application in the microcircuits working on ultrahigh frequencies. Thin films from monocrystals are effectively used as waveguide's systems. At present, artificial anisotropic materials are especially actual for the design of microwave integrated circuits and optical devices. The technology advances are making the production of substrates, dielectric anisotropic films and anisotropic material filling more and more convenient.

Due to the complexity caused by the parameter tensors, the plane wave expansion (Born \& Wolf, 1999) is often used in the analysis of anisotropic media. The standard mathematical technique for treating propagation through a homogeneous anisotropic medium is to investigate the independent time-harmonic plane wave solutions of Maxwell's equations. And consequently, the Fourier transform is widely applied (Chen, 1983; Kong, 1986; Ren, 1993; Uzunoglu et al., 1985).

The radiation field of a dipole in a anisotropic medium is considered in greater detail and devised by (Bunkin, 1957; Clemmow, 1963a;b; Kogelnik \& Motz, 1963). It is shown (Clemmow, 1963a;b) that each such field is related by a simple scaling procedure to a corresponding vacuum field. The vacuum field is expressed as the superposition of a transverse magnetic field, in which the magnetic vector is everywhere perpendicular to the axis of symmetry of the anisotropic medium, and a coplanar transverse electric field; and different scaling is applied separately to each partial field. But because of the inevitable complication of any such general analysis it also seemed desirable to isolate the simplest non-trivial case.

Using these methods, (Uzunoglu et al., 1985) found the solution of the vector wave equation in cylindrical coordinates for a gyroelectric medium. (Ren, 1993) continued that work for spherical coordinates in a similar procedure and obtained spherical wave functions and dyadic Green's functions in gyroelectric media. The dyadic Green's functions for various kinds of anisotropic media with different structures have been studied by many authors (Barkeshli, 1993; Cottis, 1995; Lee \& Kong, 1983; Weiglhofer, 1990; 1993). The problems, however, are mostly analyzed in spectral domain in terms of Fourier transform, due to the 
difficulty of finding the expansion of the dyadic Green's functions in terms of vector wave functions for anisotropic media. It shows the necessity of better characterizing the anisotropic media and producing more realistic models for the components that use them.

The purpose of this chapter is to obtain explicit expressions for the stationary problem of the field produced by a given distribution of external currents in an infinite homogeneous uniaxial anisotropic medium without using the scaling procedure to a corresponding vacuum field and the dyadic Green's functions.

This chapter is organized as follows: in the following section, section 2, we present the method of generalized function to solve the Maxwell's equations for isotropic media. To demonstrate the method we deduce the general solutions of Maxwell's equations by means of the unique Green's function. Interrelation between the Green's function and electrodynamic potentials and also the Hertz potential is shown. Some forms of the Green's function for lower dimension are presented. In section 3, the method of generalized functions is employed for solving the problem of uniaxial crystals. We introduce there a stationary electromagnetic field $(\boldsymbol{E}, \boldsymbol{H})$ of frequency $\boldsymbol{\omega}$ and formulate the problem. We use the generalized method of the Fourier transformation for solving the matrix form of Maxwell's equations. Radiation of electric and magnetic dipole is considered and their directivity diagrams are obtained. In section 4, we adduce all points of section 3 only for magnetic media. Finally, in section 5 conclusions and future related research are presented. In this chapter, we can note the following main results. The fundamental solutions of a system of Maxwell's equations for uniaxial anisotropic media are obtained. Due to the fundamental solutions, general exact expression of an electromagnetic field in boundless uniaxial crystal is obtained in the vector type by the method of generalized functions. The results are valid for any values of the elements of the permeability tensor, as well as for sources of the electromagnetic waves described by discontinuous and singular functions. In particular, the solutions for elementary electric and magnetic dipoles have been deduced. Through the use of the expressions for current density of the point magnetic and electric dipoles using delta-function representations, the formulae for the radiated electromagnetic waves, as well as the corresponding radiation patterns, are derived. The obtained solution in the anisotropic case yields the well-known solutions for the isotropic case as a limiting case. Validity of the solutions have been checked up on balance of energy by integration of energy flow on sphere. The numerical calculation of the solution of Maxwell's equations shows that it satisfies the energy conservation law.

By means of the method of generalized functions it is possible to represent the electromagnetic field in the integral form with respect to their values on an arbitrary closed surface. Subsequently analytical solutions of Maxwell's equations obtained in this chapter for unbounded anisotropic media allow to construct the integral equations for solving the class of corresponding boundary problems. The obtained solutions can be easily generalized taking into account magnetic currents. Because electric and magnetic currents are independent, it makes it possible to decompose a solution on separate independent solutions. The solutions can be also represented with the help of vector potentials for the corresponding fundamental solutions.

\section{Method of generalized functions}

To present the method of generalized functions (Alekseyeva \& Sautbekov, 1999; 2000) we shall consider stationary Maxwell's equations for isotropic media 


$$
\left\{\begin{array}{l}
\nabla \times \boldsymbol{H}+i \omega \boldsymbol{D}=\boldsymbol{j} \\
\nabla \times \boldsymbol{E}-i \omega \boldsymbol{B}=0 .
\end{array}\right.
$$

The linear relation between the induction and the intensity of electric field in isotropic dielectric mediums is:

$$
\boldsymbol{D}=\varepsilon \varepsilon_{0} E
$$

and the vector of magnetic induction is given by:

$$
\mathbf{B}=\mu \mu_{0} \boldsymbol{H}
$$

$\mu, \varepsilon$ are the relative magnetic and dielectric permeability respectively, $E$ and $H$ are the intensity of electric and magnetic fields respectively, and $j$ is vector of current density.

We will present the system (1) in the matrix form:

$$
\mathbf{M U}=\mathbf{J}
$$

where

$$
\begin{gathered}
\mathbf{M}=\left(\begin{array}{cc}
i \omega \varepsilon_{0} \varepsilon \boldsymbol{I} & \boldsymbol{G}_{0} \\
\boldsymbol{G}_{0} & -i \omega \mu_{0} \mu \boldsymbol{I}
\end{array}\right), \quad \boldsymbol{G}_{0}=\left(\begin{array}{ccc}
0 & -\partial_{z} & \partial_{y} \\
\partial_{z} & 0 & -\partial_{x} \\
-\partial_{y} & \partial_{x} & 0
\end{array}\right), \\
\mathbf{U}=\left(\begin{array}{l}
\boldsymbol{E} \\
\boldsymbol{H}
\end{array}\right), \quad \mathbf{J}=\left(\begin{array}{c}
\boldsymbol{j} \\
\boldsymbol{0}
\end{array}\right), \quad \boldsymbol{E}=\left(\begin{array}{l}
E_{x} \\
E_{y} \\
E_{z}
\end{array}\right), \quad \boldsymbol{H}=\left(\begin{array}{l}
H_{x} \\
H_{y} \\
H_{z}
\end{array}\right), \quad j=\left(\begin{array}{l}
j_{x} \\
j_{y} \\
j_{z}
\end{array}\right), \quad \boldsymbol{\quad}=\left(\begin{array}{l}
0 \\
0 \\
0
\end{array}\right),
\end{gathered}
$$

$\omega$ is the constant frequency of electromagnetic field, $\mathbf{M}$ is Maxwell's operator, $\boldsymbol{I}$ is a identity matrix $3 \times 3$.

A method generalized functions based on the theory of the generalized function of the Fourier transformation is used for solving the matrix equation (4) (Vladimirov, 2002):

$$
\tilde{\boldsymbol{E}}(\boldsymbol{k})=\mathbf{F}[\boldsymbol{E}(\boldsymbol{r})]=\int_{\mathbb{R}^{3}} \boldsymbol{E}(\boldsymbol{r}) \exp (-i \boldsymbol{k} \boldsymbol{r}) d V
$$

where

$$
\begin{gathered}
\boldsymbol{E}(\boldsymbol{r})=\mathbf{F}^{-1}[\tilde{\boldsymbol{E}}(\boldsymbol{k})]=\int_{\mathbb{R}^{3}} \tilde{\boldsymbol{E}}(\boldsymbol{k}) \exp (i \boldsymbol{k} \boldsymbol{r}) \frac{d^{3} k}{(2 \pi)^{3}}, \\
\boldsymbol{k}=\left(k_{x}, k_{y}, k_{z}\right), \quad d^{3} k=d k_{x} k_{y} k_{z}, \quad d V=d x d y d z, \quad \boldsymbol{r}=(x, y, z) .
\end{gathered}
$$

By means of direct Fourier transformation we will write down the system of equations (1) or (4) in matrix form:

$$
\begin{gathered}
\tilde{\mathbf{M}} \tilde{\mathbf{U}}=\tilde{\mathbf{J}}, \\
\tilde{\mathbf{M}}=\left(\begin{array}{cc}
i \varepsilon_{0} \varepsilon \omega \boldsymbol{I} & \tilde{\boldsymbol{G}_{0}} \\
\tilde{\boldsymbol{G}_{0}} & -i \mu_{0} \mu \omega \boldsymbol{I}
\end{array}\right), \quad \tilde{\boldsymbol{G}_{0}}=i\left(\begin{array}{ccc}
0 & -k_{z} & k_{y} \\
k_{z} & 0 & -k_{x} \\
-k_{y} & k_{x} & 0
\end{array}\right) .
\end{gathered}
$$

The solution of the problem is reduced to determination of the system of the linear algebraic equations relative to Fourier-components of the fields, where $\tilde{\mathbf{U}}$ is defined by means of inverse 
$\operatorname{matrix} \tilde{\mathbf{M}}^{-1}$ :

$$
\tilde{\mathbf{U}}=\tilde{\mathbf{M}}^{-1} \tilde{\mathbf{J}} .
$$

By introducing new function according to

$$
\tilde{\psi}_{0}=\frac{1}{k_{0}^{2}-k^{2}}
$$

we define the inverse matrix:

$$
\tilde{\mathbf{M}}^{-1}=\tilde{\psi}_{0}\left(\begin{array}{cc}
-\left(\varepsilon_{0} \varepsilon\right)^{-1} \tilde{\boldsymbol{G}}_{1} & -\tilde{\boldsymbol{G}}_{0} \\
-\tilde{\boldsymbol{G}}_{0} & \left(\mu_{0} \mu\right)^{-1} \tilde{\boldsymbol{G}}_{1}
\end{array}\right),
$$

where

$$
\begin{gathered}
\tilde{G}_{1}=\frac{1}{i \omega}\left(\begin{array}{ccc}
k_{1}^{2}-k_{0}^{2} & k_{1} k_{2} & k_{1} k_{3} \\
k_{1} k_{2} & k_{2}^{2}-k_{0}^{2} & k_{2} k_{3} \\
k_{1} k_{3} & k_{2} k_{3} & k_{3}^{2}-k_{0}^{2}
\end{array}\right), \\
k_{0} \equiv \omega \sqrt{\varepsilon_{0} \varepsilon \mu_{0} \mu}, \quad k^{2}=k_{x}^{2}+k_{y}^{2}+k_{z}^{2} .
\end{gathered}
$$

By considering the inverse Fourier transformation

$$
\mathbf{M}^{-1}=\mathrm{F}^{-1}\left[\tilde{\mathbf{M}}^{-1}\right], \quad \mathbf{J}=\mathrm{F}^{-1}[\tilde{\mathbf{J}}], \quad \mathbf{U}=\mathrm{F}^{-1}[\tilde{\mathbf{U}}]
$$

and using the property of convolution:

$$
\mathrm{F}^{-1}\left[\tilde{\mathbf{M}}^{-1} \tilde{\mathbf{J}}\right]=\mathbf{M}^{-1} * \mathbf{J},
$$

where symbol "*" denotes the convolution on coordinates $x, y, z$, it is possible to get the solution of the Maxwell equations (4) as:

$$
\mathbf{U}=\mathbf{M}^{-1} * \mathbf{J},
$$

where

$$
\mathbf{M}^{-1}=\left(\begin{array}{cc}
-\left(\varepsilon_{0} \varepsilon\right)^{-1} G_{1} & -G_{0} \\
-G_{0} & \left(\mu_{0} \mu\right)^{-1} G_{1}
\end{array}\right) \psi_{0}, \quad G_{1}=\frac{-1}{i \omega}\left(\begin{array}{ccc}
\frac{\partial^{2}}{\partial x^{2}}+k_{0}^{2} & \frac{\partial^{2}}{\partial x \partial y} & \frac{\partial^{2}}{\partial x \partial z} \\
\frac{\partial^{2}}{\partial x \partial y} & \frac{\partial^{2}}{\partial y^{2}}+k_{0}^{2} & \frac{\partial^{2}}{\partial y \partial z} \\
\frac{\partial^{2}}{\partial x \partial z} & \frac{\partial^{2}}{\partial y \partial z} & \frac{\partial^{2}}{\partial z^{2}}+k_{0}^{2}
\end{array}\right)
$$

or

$$
\left(\begin{array}{c}
\boldsymbol{E} \\
\boldsymbol{H}
\end{array}\right)=\left(\begin{array}{c}
\left(i \varepsilon_{0} \varepsilon \omega\right)^{-1}\left(\nabla \nabla+k_{0}^{2}\right)\left(\boldsymbol{j} * \psi_{0}\right) \\
-\nabla \times\left(\boldsymbol{j} * \psi_{0}\right)
\end{array}\right)=\left(\begin{array}{c}
\left(\varepsilon_{0} \varepsilon\right)^{-1} \nabla \rho * \psi_{0}-i \mu_{0} \mu \omega \psi_{0} * j \\
j \times * \nabla \psi_{0}
\end{array}\right),
$$

according to the charge conservation low

$$
\nabla j-i \omega \rho=0 .
$$

Here $\rho$ is charge density, $\psi_{0}$ is the Green's function or a fundamental solution of the Helmholtz operator for isotropic medium (Vladimirov, 2002):

$$
\psi_{0}=\mathrm{F}^{-1}\left[\tilde{\psi}_{0}\right]=-\frac{1}{4 \pi} \frac{e^{i k_{0} r}}{r}, \quad r=\sqrt{x^{2}+y^{2}+z^{2}}
$$


which satisfy the equation:

$$
\left(\triangle+k_{0}^{2}\right) \psi_{0}=\delta(\boldsymbol{r})
$$

On the other hand, we can obtain Eqn. (23), after application of the inverse Fourier transformation from Eqn. (12).

Thus, the general solution of the system of stationary Maxwell's equations for a three-dimensional unbounded isotropic media was deduced by means of solely one fundamental solution $\psi_{0}$. Hence, the solution preserves the same form concerning the fundamental solution $\psi_{0}$ for two-dimensional and one-dimensional problems. The useful forms of the fundamental solutions are adduced below by means of Fourier transformations:

$$
\begin{gathered}
\psi_{0}\left(x, y, k_{z}, \omega\right)=-\frac{i}{4} \mathrm{H}_{0}^{(1)}\left(\sqrt{k_{0}^{2}-k_{z}^{2}} \sqrt{x^{2}+y^{2}}\right) \quad \text { (two-dimensional case) } \\
\psi_{0}\left(k_{x}, y, k_{z}, \omega\right)=\frac{\exp (i h|y|)}{2 i h}, \quad h=\sqrt{k_{0}^{2}-k_{x}^{2}-k_{z}^{2}}-\text { const (one-dimensional case). }
\end{gathered}
$$

\subsection{Electrodynamic potentials and Hertz vector}

It should be noted that all electrodynamic quantities of isotropic media can be expressed by function $\psi_{0}$, including the electrodynamic potentials and Hertz vector. By designating the scalar potential $\varphi$ and vector potential $A$ :

$$
\varphi=-\left(\varepsilon_{0} \varepsilon\right)^{-1} \psi_{0} * \rho, \quad \boldsymbol{A}=-\mu_{0} \mu \psi_{0} * j,
$$

the solution (18) or (20) can be presented in known form by vector potential

$$
\boldsymbol{E}=-\nabla \varphi+i \omega \boldsymbol{A}, \quad \boldsymbol{H}=\left(\mu_{0} \mu\right)^{-1} \nabla \times \boldsymbol{A} .
$$

It should be noted that physical sense of Lorentz gauge of potentials consists in the charge conservation law (21), indeed

$$
\nabla A-i \omega \varepsilon_{0} \varepsilon \mu_{0} \mu \varphi=-\mu_{0} \mu \psi_{0} *(\nabla j-i \omega \rho)=0 .
$$

Similarly, by designating Hertz vector

$$
\Pi=\left(i \omega \varepsilon_{0} \varepsilon\right)^{-1} j * \psi_{0}
$$

solution (18) can be written as

$$
\begin{gathered}
\boldsymbol{E}=\left(\nabla \nabla+k_{0}^{2}\right) \boldsymbol{\Pi}=\nabla \times \nabla \times \boldsymbol{\Pi}+\left(i \varepsilon_{0} \varepsilon \omega\right)^{-1} \boldsymbol{j}, \\
\boldsymbol{H}=-i \varepsilon_{0} \varepsilon \omega \nabla \times \boldsymbol{\Pi} .
\end{gathered}
$$

It is easy to take notice that the relation between the electrodynamic potentials and Hertz potential is

$$
A=-i \omega \varepsilon_{0} \varepsilon \mu_{0} \mu \Pi, \quad \varphi=-\nabla \Pi .
$$




\section{The generalized solutions of Maxwell equations for the uniaxial crystal}

The exact analytical solutions of Maxwell's equations are constructed by means of method of generalized functions in vector form for unlimited uniaxial crystals (Sautbekov et al., 2008). The fundamental solutions of a system of Maxwell's equations for uniaxial crystals are obtained. The solution of the problem was analyzed in Fourier space and closed form analytical solutions were derived in Section (3.2), above. Then, when the current distribution is defined in such a medium, the corresponding radiated electric and magnetic fields can be calculated anywhere in space. In particular, the solutions for elementary electric dipoles have been deduced in Section(3.3), and the radiation patterns for Hertz radiator dipole are represented. The governing equations and radiation pattern in the case of an unbounded isotropic medium were obtained as a special case. Validity of the solutions have been checked up on balance of energy by integration of energy flow on sphere.

\subsection{Statement of the problem}

The electric and magnetic field strengths satisfy system of stationary the Maxwell's equations (1), which is possible to be presented in matrix form (4), where

$$
\mathbf{M}=\left(\begin{array}{cc}
-i \omega \varepsilon_{0} \hat{\varepsilon} & G_{0} \\
G_{0} & i \mu_{0} \mu \boldsymbol{I}
\end{array}\right), \quad \hat{\varepsilon}=\left(\begin{array}{ccc}
\varepsilon_{1} & 0 & 0 \\
0 & \varepsilon & 0 \\
0 & 0 & \varepsilon
\end{array}\right) .
$$

The relation between the induction and the intensity of electric field in anisotropic dielectric mediums is:

$$
\boldsymbol{D}=\hat{\varepsilon} \varepsilon_{0} E .
$$

If we choose a frame in main axes of dielectric tensor, the constitutive equation will be written as:

$$
D_{x}=\varepsilon_{1} \varepsilon_{0} E_{x}, \quad D_{y}=\varepsilon \varepsilon_{0} E_{y}, \quad D_{z}=\varepsilon \varepsilon_{0} E_{z} .
$$

The elements of the dielectric permeability tensor $\hat{\varepsilon}$ correspond to a one-axis crystal, moreover the axis of the crystal is directed along axis $x$. Moreover, it is required to define the intensities of the electromagnetic field $\boldsymbol{E}, \boldsymbol{H}$ in the space of generalized function.

\subsection{Problem solution}

By means of direct Fourier transformation, we write down the system of equations in matrix form (9). The solution of the problem is reduced to determination of the system of the linear algebraic equations relative to Fourier-components of the fields, where $\tilde{\mathbf{U}}$ is defined by means of inverse matrix $\tilde{\mathbf{M}}^{-1}$. By introducing new functions according to $\tilde{\psi}_{0}$ (12) and

$$
\tilde{\psi}_{1}=\left(k_{n}^{2}-\frac{\varepsilon_{1}}{\varepsilon} k_{x}^{2}-k_{y}^{2}-k_{z}^{2}\right)^{-1}, \quad \tilde{\psi}_{2}=\left(\frac{\varepsilon_{1}}{\varepsilon}-1\right) \tilde{\psi}_{1} \tilde{\psi}_{0}
$$

the components of the electromagnetic field after transformations in Fourier space can be written as follows:

$$
\left\{\begin{array}{l}
\tilde{E}_{x}=\left(i \varepsilon \varepsilon_{0} \omega\right)^{-1}\left(k_{0}^{2} \tilde{j}_{x}-k_{x} \boldsymbol{k} \tilde{j}\right) \tilde{\psi}_{1}, \\
\tilde{E}_{y}=\left(i \varepsilon \varepsilon_{0} \omega\right)^{-1}\left(k_{0}^{2} \tilde{\psi}_{0} \tilde{j}_{y}-k_{y} \tilde{\psi}_{1} \boldsymbol{k} \tilde{j}-k_{0}^{2} k_{y} \tilde{\psi}_{2} \boldsymbol{k} \tilde{j}_{\perp}\right), \\
\tilde{E}_{z}=\left(i \varepsilon \varepsilon_{0} \omega\right)^{-1}\left(k_{0}^{2} \tilde{\psi}_{0} \tilde{j}_{z}-k_{z} \tilde{\psi}_{1} \boldsymbol{k} \tilde{j}-k_{0}^{2} k_{z} \tilde{\psi}_{2} \boldsymbol{k} \tilde{j}_{\perp}\right),
\end{array}\right.
$$




$$
\left\{\begin{array}{l}
\tilde{H}_{x}=-i \tilde{\psi}_{0}(\boldsymbol{k} \times \tilde{\boldsymbol{j}})_{x}, \\
\tilde{H}_{y}=-i\left(k_{z} j_{x} \tilde{\psi}_{1}-k_{x} k_{z} \tilde{\psi}_{2} \boldsymbol{k} \tilde{j}_{\perp}+k_{x} \tilde{j}_{z} \tilde{\psi}_{0}\right), \\
\tilde{H}_{z}=-i\left(k_{x} \tilde{j}_{y} \tilde{\psi}_{0}+k_{y} \tilde{j}_{x} \tilde{\psi}_{1}+k_{x} k_{y} \tilde{\psi}_{2} \boldsymbol{k} \tilde{j}_{\perp}\right),
\end{array}\right.
$$

where,

$$
\tilde{j}=\tilde{j}_{\perp}+\tilde{j}_{0}, \quad \tilde{j}_{0}=\left(\tilde{j}_{x}, 0,0\right), \quad \tilde{j}_{\perp}=\left(0, \tilde{j}_{y}, \tilde{j}_{z}\right), \quad k_{n}^{2}=k_{0}^{2} \varepsilon_{1} / \varepsilon,
$$

that $k_{n}$ is the propagation constant along the axis of the crystal ( $x$-axis), and $k_{0}$ is the propagation constant along the $y$ and $z$ axis. It is possible to present the electromagnetic fields in vector type:

$$
\begin{gathered}
\tilde{\boldsymbol{E}}=-\frac{i}{\varepsilon_{0} \varepsilon \omega}\left(k_{0}^{2}\left\{\tilde{\boldsymbol{j}}_{\perp} \tilde{\psi}_{0}+\tilde{\boldsymbol{j}}_{0} \tilde{\psi}_{1}+\left(k_{x} \boldsymbol{k} \tilde{\boldsymbol{j}}_{\perp}-\boldsymbol{k}\left(\boldsymbol{k} \tilde{\boldsymbol{j}}_{\perp}\right)\right) \tilde{\psi}_{2}\right\}-\boldsymbol{k}(\boldsymbol{k} \tilde{\boldsymbol{j}}) \tilde{\psi}_{1}\right), \\
\tilde{\boldsymbol{H}}=-i \boldsymbol{k} \times\left(\tilde{\boldsymbol{j}}_{\perp} \tilde{\psi}_{0}+\tilde{\boldsymbol{j}}_{0} \tilde{\psi}_{1}+\boldsymbol{k}_{x}\left(\boldsymbol{k} \tilde{\boldsymbol{j}}_{\perp}\right) \tilde{\psi}_{2}\right), \quad \boldsymbol{k}_{x}=\mathbf{e}_{x}\left(\mathbf{e}_{x} \boldsymbol{k}\right),
\end{gathered}
$$

where $\mathbf{e}_{x}$ is the unit vector along $x$-axis. Using the property of convolution and by considering the inverse Fourier transformation it is possible to get the solution of the Maxwell equations in the form of the sum of two independent solutions:

$$
E=E_{1}+E_{2}, \quad H=H_{1}+H_{2} .
$$

The first of them, fields $\boldsymbol{E}_{1}$ and $\boldsymbol{H}_{1}$, is defined by one Green function $\psi_{1}$ and the density of the current $j_{0}$ along the axis of the crystal:

$$
\left\{\begin{array}{l}
\boldsymbol{E}_{1}=\left(i \varepsilon_{0} \varepsilon \omega\right)^{-1}\left(\nabla \nabla+k_{0}^{2}\right)\left(\psi_{1} * j_{0}\right), \\
\boldsymbol{H}_{1}=-\nabla \times\left(\psi_{1} * \boldsymbol{j}_{0}\right),
\end{array}\right.
$$

where $r^{\prime}=\sqrt{x^{2} \varepsilon / \varepsilon_{1}+y^{2}+z^{2}}$ and

$$
\psi_{1}=-\frac{1}{4 \pi} \sqrt{\frac{\varepsilon}{\varepsilon_{1}}} \frac{e^{i k_{n} r^{\prime}}}{r^{\prime}} .
$$

The second solution, fields $\boldsymbol{E}_{2}$ and $\boldsymbol{H}_{2}$, can be written by using the component of the density of the current $j_{\perp}$ perpendicular to axis $x$ and Green functions $\psi_{0}, \psi_{1}$ and $\psi_{2}$ :

$$
\left\{\begin{array}{c}
\boldsymbol{E}_{2}=\left(i \varepsilon_{0} \varepsilon \omega\right)^{-1}\left(k_{0}^{2}\left(j_{\perp} * \psi_{0}+\nabla_{\perp} \nabla j_{\perp} * \psi_{2}\right)+\nabla \nabla\left(j_{\perp} * \psi_{1}\right)\right), \\
\boldsymbol{H}_{2}=-\nabla \times\left(j_{\perp} * \psi_{0}-\mathbf{e}_{x} \frac{\partial}{\partial x} \nabla j_{\perp} * \psi_{2}\right), \\
\psi_{2}=\left(\frac{\varepsilon_{1}}{\varepsilon}-1\right) \psi_{0} * \psi_{1}, \\
\nabla_{\perp} \equiv \nabla-\mathbf{e}_{x} \frac{\partial}{\partial x} .
\end{array}\right.
$$

We note here that the function $\psi_{0}(22)$ is a fundamental solution of the Helmholtz operator for isotropic medium, while $\psi_{1}$ (39) corresponds to the functions $\psi_{0}$ for the space deformed along 
the axis of the crystal. Furthermore, the following useful identities are valid:

$$
\begin{gathered}
\mathrm{F}^{-1}\left[\tilde{\psi}_{0}\left(k_{0}^{2}-k_{x}^{2}\right)^{-1}\right]=\psi_{0} * \mathrm{~F}^{-1}\left[\left(k_{0}^{2}-k_{x}^{2}\right)^{-1}\right]= \\
-\frac{i}{8 \pi k_{0}}\left(e^{i k_{0} x}\left(\mathrm{Ci}\left(k_{0}(r-x)\right)+i \operatorname{si}\left(k_{0}(r-x)\right)\right)+e^{-i k_{0} x}\left(\mathrm{Ci}\left(k_{0}(r+x)\right)+i \operatorname{si}\left(k_{0}(r+x)\right)\right)\right), \\
\mathrm{F}^{-1}\left[\tilde{\psi}_{1}\left(k_{0}^{2}-k_{x}^{2}\right)^{-1}\right]=\psi_{1} * \mathrm{~F}^{-1}\left[\left(k_{0}^{2}-k_{x}^{2}\right)^{-1}\right]=-\frac{i}{8 \pi k_{0}}\left(e ^ { i k _ { 0 } x } \left(\operatorname{Ci}\left(k_{n} r^{\prime}-k_{0} x\right)+\right.\right. \\
\left.\left.i \operatorname{si}\left(k_{n} r^{\prime}-k_{0} x\right)\right)+e^{-i k_{0} x}\left(\operatorname{Ci}\left(k_{n} r^{\prime}+k_{0} x\right)+i \operatorname{si}\left(k_{n} r^{\prime}+k_{0} x\right)\right)\right), \\
\mathrm{F}^{-1}\left[\tilde{\psi}_{2}\right]=\mathrm{F}^{-1}\left[\tilde{\psi}_{0}\left(k_{0}^{2}-k_{x}^{2}\right)^{-1}\right]-\mathrm{F}^{-1}\left[\tilde{\psi}_{1}\left(k_{0}^{2}-k_{x}^{2}\right)^{-1}\right] .
\end{gathered}
$$

Therefore, by also using (44), (45) above we find that the function $\psi_{2}$ is given by:

$$
\begin{array}{r}
\psi_{2}=\frac{1}{8 \pi k_{0} i}\left(e^{i k_{0} x}\left(\operatorname{Ci}\left(k_{0}(r-x)\right)+i \operatorname{si}\left(k_{0}(r-x)\right)\right)+e^{-i k_{0} x}\left(\operatorname{Ci}\left(k_{0}(r+x)\right)+i \operatorname{si}\left(k_{0}(r+x)\right)\right)-\right. \\
\left.e^{i k_{0} x}\left(\operatorname{Ci}\left(k_{n} r^{\prime}-k_{0} x\right)+i \operatorname{si}\left(k_{n} r^{\prime}-k_{0} x\right)\right)-e^{-i k_{0} x}\left(\operatorname{Ci}\left(k_{n} r^{\prime}+k_{0} x\right)+i \operatorname{si}\left(k_{n} r^{\prime}+k_{0} x\right)\right)\right),
\end{array}
$$

where integral cosine and integral sine functions are defined by the following formulae:

$$
\operatorname{Ci}(z)=\gamma+\ln (z)+\int_{0}^{z} \frac{\cos (t)-1}{t} d t, \quad \operatorname{si}(z)=\int_{0}^{z} \frac{\sin (t)}{t} d t-\frac{\pi}{2}
$$

and Euler constant $\gamma=0,5772$.

Solutions (19), (20) and (22) can be also represented with the help of vector potentials $A_{0}, A_{1}$ and $A_{2}$ as follows:

$$
\left\{\begin{array}{l}
\boldsymbol{E}=i \omega\left(\boldsymbol{A}_{0}+\mathbf{e}_{x}\left(\mathbf{e}_{x} \boldsymbol{A}_{1}\right)+\nabla_{\perp} \nabla \boldsymbol{A}_{2}+\frac{1}{k_{0}^{2}} \nabla \nabla \boldsymbol{A}_{1}\right), \\
\boldsymbol{H}=\frac{1}{\mu \mu_{0}} \nabla \times\left(\boldsymbol{A}_{0}+\mathbf{e}_{x}\left(\mathbf{e}_{x} \boldsymbol{A}_{1}\right)-\mathbf{e}_{x} \frac{\partial}{\partial x} \nabla \boldsymbol{A}_{2}\right) .
\end{array}\right.
$$

The vector potentials $A_{0}, A_{1}$ and $A_{2}$ satisfy the following equations:

$$
\begin{gathered}
\left(\triangle+k_{0}^{2}\right) A_{0}=-\mu \mu_{0} j_{\perp}, \quad\left(\triangle^{\prime}+k_{n}^{2}\right) A_{1}=-\mu \mu_{0} j, \\
\left(\triangle+k_{0}^{2}\right)\left(\triangle^{\prime}+k_{n}^{2}\right) A_{2}=-\mu \mu_{0}\left(\varepsilon_{1} / \varepsilon-1\right) j_{\perp},
\end{gathered}
$$

where $\triangle$ is the Laplace operator, the prime in $\triangle$ corresponds a replacement $x \rightarrow x \varepsilon / \varepsilon_{1}$. The solutions of the equations (49), (50) can be written as follows:

$$
A_{0}=-\mu \mu_{0} \cdot j_{\perp} * \psi_{0}, \quad A_{1}=-\mu \mu_{0} \cdot j * \psi_{1}, \quad A_{2}=-\mu \mu_{0} \cdot j_{\perp} * \psi_{2} .
$$




\subsection{Hertz radiator in one-axis crystals}

On the basis of the results obtained above, we shall consider the radiation of the electric Hertzian dipole in unbounded one-axis crystals. The point dipole moment is given by

$$
p=n p_{e} \exp (-i \omega t)
$$

where $p_{e}$ is a constant, $n$ is a unit vector parallel to the direction of the dipole moment, and the current density is defined by means of Dirac delta-function :

$$
j=-i \omega p \delta(r), \quad p=p_{0}+p_{\perp} .
$$

The last formula of current density which follows from the expression of charge density for the point dipole is given by:

$$
\rho=-(p \nabla) \delta(\boldsymbol{r})
$$

and also the charge conservation law (21).

Furthermore, the expression of the radiated electromagnetic field for electric Hertzian dipole will take the following form, when the direction of the dipole moment $p_{0}$ is parallel to the axis $x$ of the crystal (Fig. 1):

$$
\left\{\begin{array}{l}
\boldsymbol{E}_{1}=-\left(\varepsilon \varepsilon_{0}\right)^{-1}\left(\nabla \nabla+k_{0}^{2}\right)\left(\psi_{1} \boldsymbol{p}_{0}\right), \\
\boldsymbol{H}_{1}=i \omega \nabla \times\left(\psi_{1} \boldsymbol{p}_{0}\right) .
\end{array}\right.
$$

Also, when the direction of the dipole moment is perpendicular to the axis $x$, we obtain (Fig. 2):

$$
\left\{\begin{array}{l}
\boldsymbol{E}_{2}=-\frac{1}{\varepsilon \varepsilon_{0}}\left(k_{0}^{2}\left(\boldsymbol{p}_{\perp} \psi_{0}+\nabla_{\perp} \nabla\left(\boldsymbol{p}_{\perp} \psi_{2}\right)\right)+\nabla \nabla\left(\boldsymbol{p}_{\perp} \psi_{1}\right)\right), \\
\boldsymbol{H}_{2}=-i \omega \nabla \times\left(\boldsymbol{p}_{\perp} \psi_{0}-\mathbf{e}_{x} \frac{\partial}{\partial x} \nabla\left(\boldsymbol{p}_{\perp} \psi_{2}\right)\right) \quad\left(\boldsymbol{p}_{0} \perp \boldsymbol{p}_{\perp}\right) .
\end{array}\right.
$$

Moreover, we note that the independent solutions (38) and (40) define the corresponding polarization of electromagnetic waves. In addition, when $\varepsilon_{1}$ tends to $\varepsilon$, from (31) it follows that the potential $\psi_{2}$ tends to zero and the well-known expressions of electromagnetic field followed from formula (38) are obtained :

$$
\begin{gathered}
\boldsymbol{E}=i \omega\left(k_{0}^{-2} \nabla \nabla+1\right) \boldsymbol{A}, \\
\boldsymbol{H}=\left(\mu \mu_{0}\right)^{-1} \nabla \times \boldsymbol{A},
\end{gathered}
$$

where the known vector potential of electromagnetic field for isotropic mediums is defined from (51) as (24):

$$
\boldsymbol{A}(\boldsymbol{r})=\frac{\mu \mu_{0}}{4 \pi} \int_{V} j\left(\boldsymbol{r}^{\prime}\right) \frac{\exp \left(i k_{0}\left|\boldsymbol{r}-\boldsymbol{r}^{\prime}\right|\right)}{\left|\boldsymbol{r}-\boldsymbol{r}^{\prime}\right|} d V
$$

The obtained generalized solutions of the Maxwell equations are valid for any values of $\varepsilon_{1}$ and $\varepsilon$, as well as for sources of the electromagnetic waves, described by discontinuous and singular functions.

Below as a specific application radiation from a Hertzian dipole in such a medium was examined and the corresponding radiation patterns were presented. 


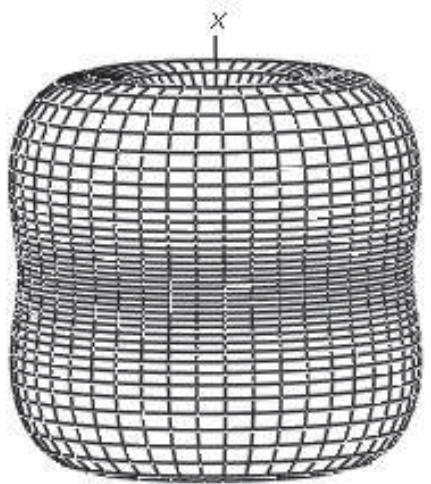

(a) Case $\varepsilon_{1}=9$

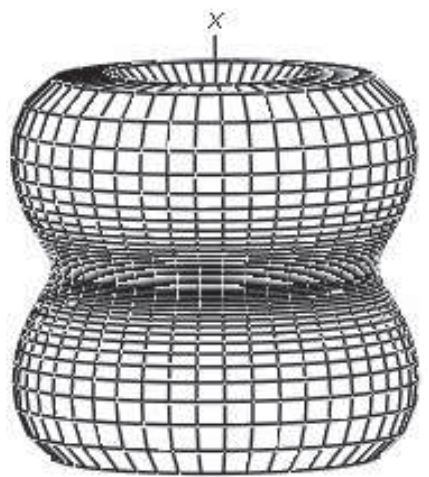

(c) Case $\varepsilon_{1}=25$

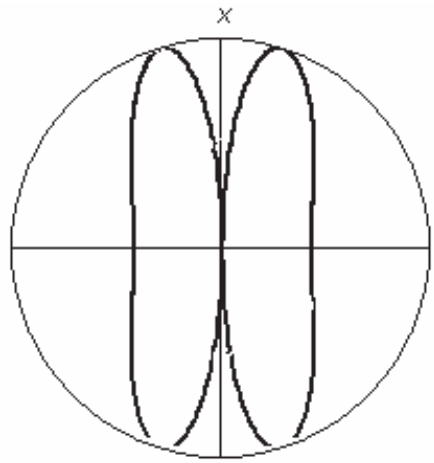

(b) View in meridian surface

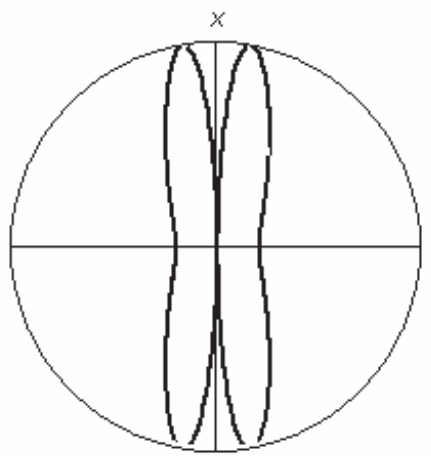

(d) View in meridian surface

Fig. 1. Directivity diagrams, the axis of dipole is parallel to axis of a crystal

It should be note that the pattern in Fig. 1 remains invariable, and independent of $r$. The radiation pattern of the Hertzian dipole in isotropic medium is shown in Fig. 3, which of course possesses rotation symmetry around $x$-axis.

Furthermore, we note here that the numerical calculation of the above solution of Maxwell equations satisfies the energy conservation law. Poynting vector

$$
<\Pi>=\frac{1}{2} \operatorname{Re}(E \times \overline{\boldsymbol{H}})
$$

is necessary to calculate time-averaged energy-flux on spherical surface

$$
\Phi=\int_{\mathcal{S}_{\text {sph }}}<\Pi_{r}>d S,
$$




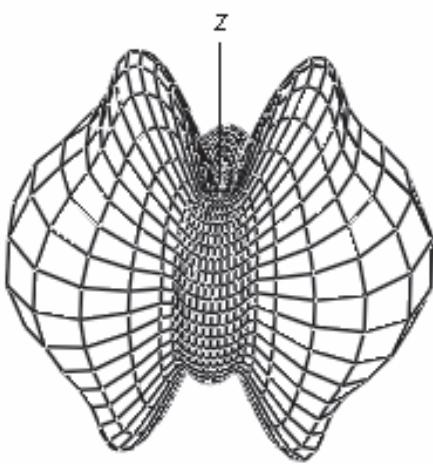

(a) Case at $r=5, \varepsilon_{1}=7$

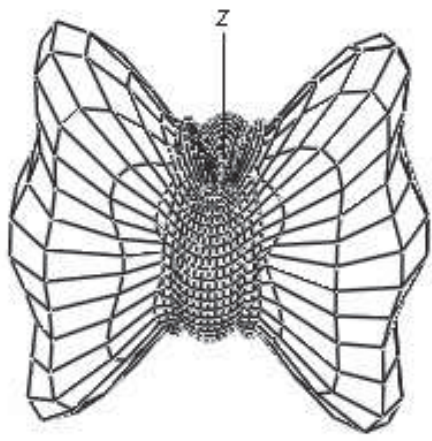

(c) Case at $r=5, \varepsilon_{1}=10$

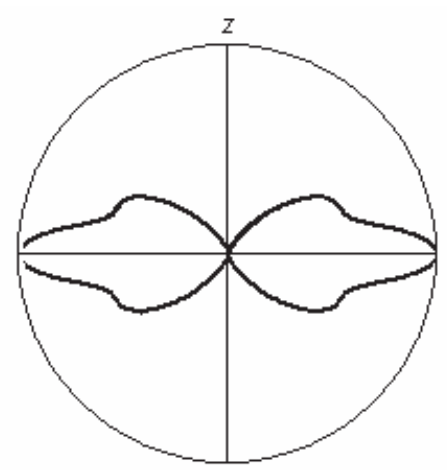

(b) View in meridian surface

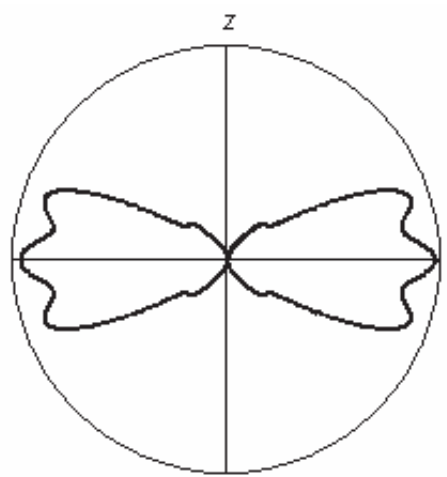

(d) View in meridian surface

Fig. 2. Directivity diagram, the axis of dipole $(z)$ is perpendicular to axis of a crystal
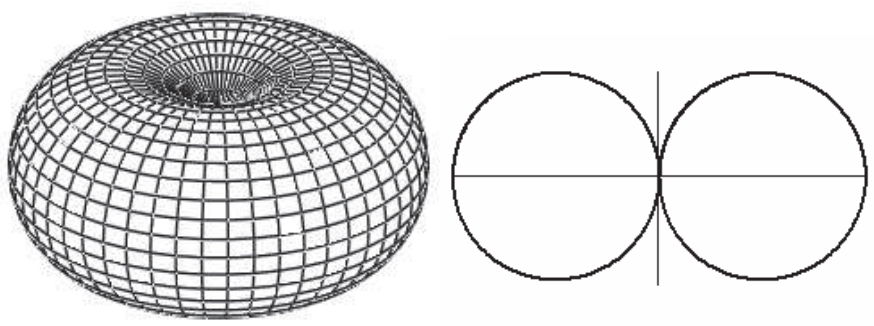

Fig. 3. Directivity diagram of the Hertzian dipole, the isotropic medium $\left(\varepsilon=\varepsilon_{1}=1\right)$

which is a constant at various values of radius of sphere, where $\bar{H}$ is complex conjugate function. 


\subsection{Directivity diagrams of the magnetic moment of a dipole in one-axis crystals}

Exact analytical solution of Maxwell's equations for radiation of a point magnetic dipole in uniaxial crystals are obtained. Directivity diagrams of radiation of a point magnetic dipole are constructed at parallel and perpendicular directions of an axis of a crystal.

On the basis of the obtained results (38) and (40), we will consider radiation of point magnetic dipole moment in an uniaxial crystal. Let's define intensity of an electromagnetic field for the concentrated magnetic dipole at a parallel and perpendicular direction to a crystal axis in the anisotropic medium and we will construct diagrams of directivity for both cases.

For a point radiator with the oscillating magnetic dipole moment

$$
\boldsymbol{m}=\boldsymbol{n} p_{m} e^{-i \omega t} \quad\left(p_{m}=\text { const }\right)
$$

the electric current density is defined by using Dirac delta-function:

$$
j=-(m \times \nabla) \delta(r) .
$$

Components of a current density (61) are:

$$
\begin{gathered}
j_{0}=\mathbf{e}_{x}\left(m_{z} \frac{\partial}{\partial y}-m_{y} \frac{\partial}{\partial z}\right) \delta(\boldsymbol{r}), \\
j_{\perp}=\mathbf{e}_{y}\left(m_{x} \frac{\partial}{\partial z}-m_{z} \frac{\partial}{\partial x}\right) \delta(\boldsymbol{r})+\mathbf{e}_{z}\left(m_{y} \frac{\partial}{\partial x}-m_{x} \frac{\partial}{\partial y}\right) \delta(\boldsymbol{r}) .
\end{gathered}
$$

It is possible to express the magnetic dipole moment $m$ in the form of the sum of two components of magnetic moment:

$$
m=m_{0}+m_{\perp}, \quad m_{0}=\mathbf{e}_{x} m_{x} .
$$

Relation between density of an electric current $j_{\perp}$ and the magnetic dipole moment $m$ in the anisotropic medium is defined from (63), in case $m=m_{0}$ :

$$
\begin{gathered}
j_{\perp}=m_{x}\left(\mathbf{e}_{y} \frac{\partial}{\partial z}-\mathbf{e}_{z} \frac{\partial}{\partial y}\right) \delta(\boldsymbol{r}), \\
\nabla \boldsymbol{j}_{\perp}=0 .
\end{gathered}
$$

\subsubsection{The parallel directed magnetic momentum}

Taking into account equality (66), from solutions (38) intensities of the electromagnetic field of the magnetic dipole moment are defined, in the case when the magnetic dipole moment $m$ is directed parallel to the crystal axis $x$ :

$$
\left\{\begin{array}{l}
\boldsymbol{E}=-i \mu_{0} \mu \omega \nabla \times\left(\psi_{0} \boldsymbol{m}_{0}\right), \\
\boldsymbol{H}=-\nabla \times \nabla \times\left(\psi_{0} \boldsymbol{m}_{0}\right) .
\end{array}\right.
$$

It is necessary to notice, that expressions (67) correspond to the equations of an electromagnetic field in isotropic medium (Fig. 4). In Fig. 4, directivity diagrams of magnetic dipole moment in the case that the magnetic moment is directed in parallel to the crystal axis are shown. The given directivity diagram coincides with the directivity diagram of a parallel 

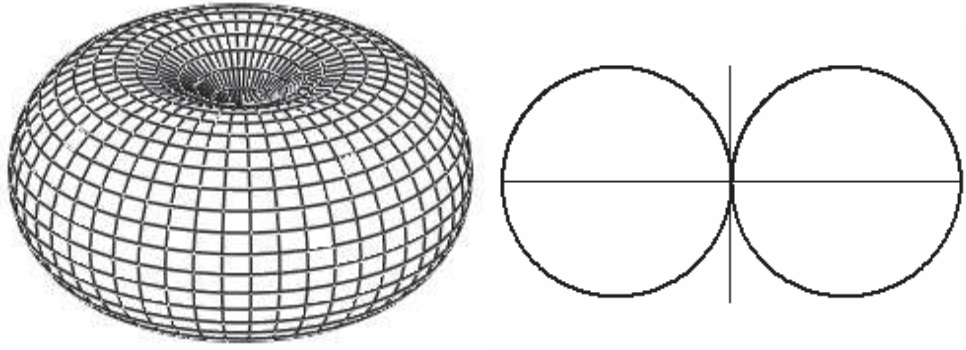

Fig. 4. Directivity diagram of the magnetic dipole, the isotropic medium $\left(\varepsilon=\varepsilon_{1}=1\right)$

directed electric dipole in isotropic medium. This diagram looks like a toroid, which axis is parallel to the dipole axis. Cross-sections of the diagram are a contour on a plane passing through an axis of the toroid. It has the shape of number 'eight' ; cross-sections perpendicular to the axis of the toroid represent circles.

\subsubsection{The perpendicular directed magnetic momentum}

Relation between density of an electric current $j$ and the magnetic dipole momentum $m_{\perp}$ is defined from expression (61), if it is directed on axis $z$ :

$$
j=m_{z}\left(\mathbf{e}_{x} \frac{\partial}{\partial y}-\mathbf{e}_{y} \frac{\partial}{\partial x}\right) \delta(\boldsymbol{r}) .
$$

For the point magnetic dipole $m_{\perp}$ which is perpendicular to crystal axes, by substituting (68) in solutions (40), we define components of field intensity (Fig. 5, Fig. 6):

$$
\begin{gathered}
\left\{\begin{array}{l}
E_{x}=-i \mu_{0} \mu \omega m_{z} \frac{\partial \psi_{1}}{\partial y} \\
E_{y}=i \mu_{0} \mu \omega m_{z} \frac{\partial}{\partial x}\left(\psi_{0}+\frac{\partial^{2} \psi_{2}}{\partial y^{2}}\right) \\
E_{z}=i \mu_{0} \mu \omega m_{z} \frac{\partial^{3} \psi_{2}}{\partial x \partial y \partial z}
\end{array}\right. \\
\left\{\begin{array}{l}
H_{x}=-m_{z} \frac{\partial^{2} \psi_{0}}{\partial x \partial z} \\
H_{y}=-m_{z} \frac{\partial^{2}}{\partial y \partial z}\left(\psi_{1}+\frac{\partial^{2} \psi_{2}}{\partial x^{2}}\right) \\
H_{z}=m_{z}\left(\frac{\partial^{2}}{\partial y^{2}}\left(\psi_{1}+\frac{\partial^{2} \psi_{2}}{\partial x^{2}}\right)+\frac{\partial^{2} \psi_{0}}{\partial x^{2}}\right)
\end{array}\right.
\end{gathered}
$$

In Figs. 5 and 6, directivity diagrams of the magnetic moment of a dipole perpendicular a crystal axis at different values of radius are shown, for two (30) values of dielectric permeability ratio, $\varepsilon_{1} / \varepsilon=9$ and $\varepsilon_{1} / \varepsilon=15$. The magnetic dipole is directed along an axis $z$ (a 


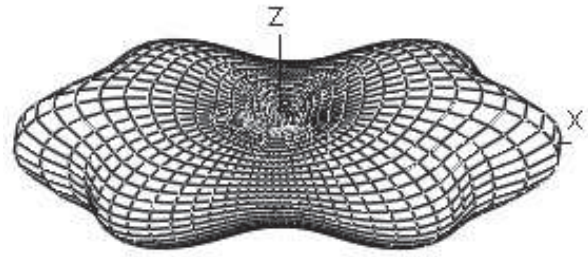

(a) Case $r=3$

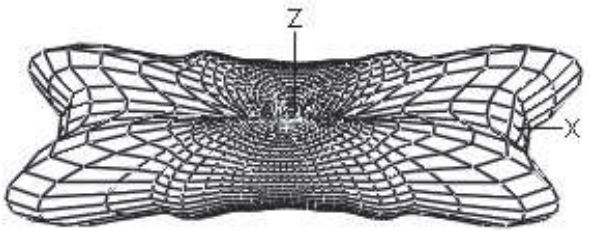

(c) Case at $r=9$

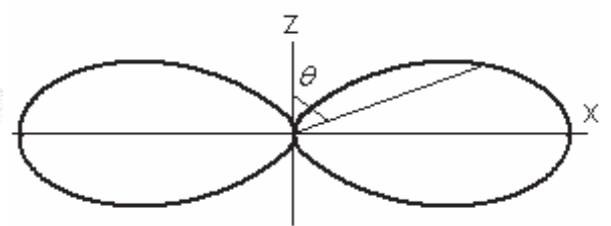

(b) View in meridian surface $r=3, \varphi=\pi / 2$

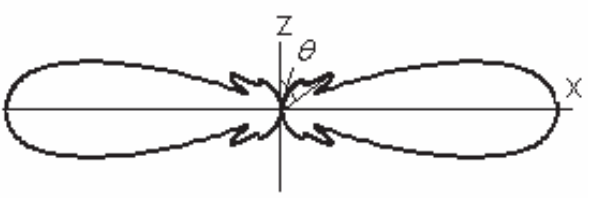

(d) View in meridian surface $r=9, \varphi=\pi / 2$

Fig. 5. Directivity diagram, the axis of magnetic dipole $(z)$ is perpendicular to axis of a crystal at $\varepsilon_{1} / \varepsilon=9$

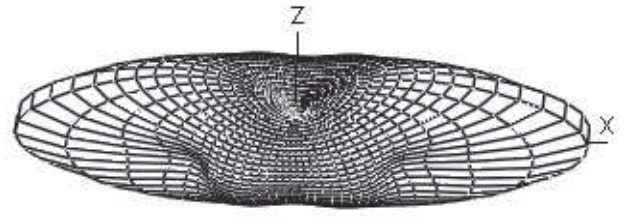

(a) Case $r=1$

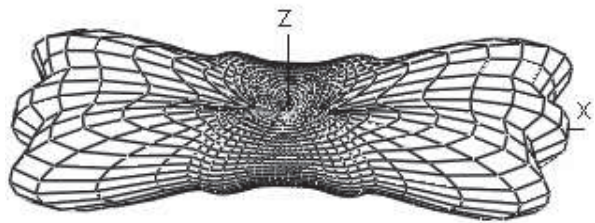

(c) Case at $r=5$

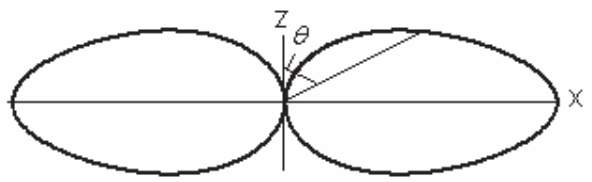

(b) View in meridian surface $r=1, \varphi=\pi / 2$

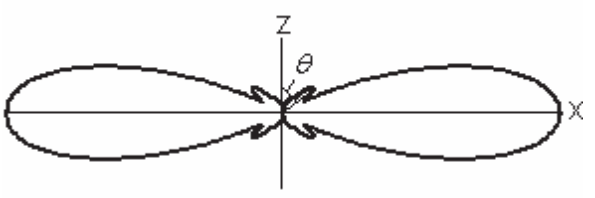

(d) View in meridian surface $r=5, \varphi=\pi / 2$

Fig. 6. Directivity diagram, the axis of magnetic dipole $(z)$ is perpendicular to axis of a crystal at $\varepsilon_{1} / \varepsilon=15$.

crystal axis - along $x$ ). As one can see from Fig. 5, that radiation in a direction of the magnetic moment does not occur, it propagates in a direction along an axis of a crystal.

Validity of solutions has been checked up on performance of the conservation law of energy. Time-averaged energy flux of energy along a surface of sphere for various values of radius was calculated for this purpose. Numerical calculations show that energy flux over the above mentioned spherical surfaces, surrounding the radiating magnetic dipole, remains constant, which means that energy conservation is preserved with high numerical accuracy. 
The obtained generalized solutions of the Maxwell equations are valid for any values of $\varepsilon_{1}$ and $\varepsilon$, and also for any kind of sources of the electromagnetic waves, described by discontinuous and singular functions.

\section{Radiation of electric and magnetic dipole antennas in magnetically anisotropic media}

The electric and magnetic field intensities satisfy the system of stationary Maxwell's equations (1) which is possible to be presented in matrix form (4), where

$$
\mathbf{M}=\left(\begin{array}{cc}
-i \omega \varepsilon_{0} \varepsilon \boldsymbol{I} & \boldsymbol{G}_{0} \\
\boldsymbol{G}_{0} & i \omega \mu_{0} \hat{\mu}
\end{array}\right), \quad \hat{\mu}=\left(\begin{array}{ccc}
\mu & 0 & 0 \\
0 & \mu & 0 \\
0 & 0 & \mu_{1}
\end{array}\right) .
$$

$\mathbf{M}$ is Maxwell's operator and $\mu$ represents the magnetic permeability.

In magnetically anisotropic media the relation between induction and intensity of the magnetic field is:

$$
\boldsymbol{B}=\mu_{0} \hat{\mu} \boldsymbol{H}, \quad B_{x}=\mu_{0} \mu H_{x}, \quad B_{y}=\mu_{0} \mu H_{y}, \quad B_{z}=\mu_{0} \mu_{1} H_{z} .
$$

The elements of the magnetic permeability tensor $\hat{\mu}$ are chosen so that the axis of anisotropy is directed along axis $z$. It is required to define the intensities of the electromagnetic field $\boldsymbol{E}, \boldsymbol{H}$ in the space of generalized functions.

\subsection{Solution of the problem}

By means of direct Fourier transformation we write down the system of the equations in matrix form (9). The solution of this problem is reduced to the solution of the system, where $\tilde{\mathbf{U}}$ is defined by means of inverse matrix $\tilde{\mathbf{M}}^{-1}$.

By introducing new functions according to

$$
\begin{gathered}
\tilde{\psi}_{1}^{m}=\frac{1}{k_{n}^{2}-k_{x}^{2}-k_{y}^{2}-\frac{\mu_{1}}{\mu} k_{z}^{2}}, \\
\tilde{\psi}_{2}^{m}=\left(\frac{\mu_{1}}{\mu}-1\right) \tilde{\psi}_{1}^{m} \tilde{\psi}_{0},
\end{gathered}
$$

the components of the electromagnetic field after transformations in spectral domain can be written as follows:

$$
\begin{aligned}
& \left\{\begin{array}{l}
\tilde{E}_{x}=\left(i \varepsilon \varepsilon_{0} \omega\right)^{-1}\left(k_{0}^{2}\left(\tilde{j}_{x} \tilde{\psi}_{0}+k_{y}(\boldsymbol{k} \times \tilde{\boldsymbol{j}})_{z} \tilde{\psi}_{2}^{m}\right)-k_{x} \boldsymbol{k} \tilde{\boldsymbol{j}} \tilde{\psi}_{0}\right), \\
\tilde{E}_{y}=\left(i \varepsilon \varepsilon_{0} \omega\right)^{-1}\left(k_{0}^{2}\left(\tilde{j}_{y} \tilde{\psi}_{0}-k_{x}(\boldsymbol{k} \times \tilde{\boldsymbol{j}})_{z} \tilde{\psi}_{2}^{m}\right)-k_{y} \boldsymbol{k} \tilde{\boldsymbol{j}} \tilde{\psi}_{0}\right), \\
\tilde{E}_{z}=\left(i \varepsilon \varepsilon_{0} \omega\right)^{-1}\left(k_{0}^{2} \tilde{j}_{z}-k_{z} \tilde{\boldsymbol{j}}\right) \tilde{\psi}_{0},
\end{array}\right. \\
& \left\{\begin{array}{l}
\tilde{H}_{x}=-i\left((\boldsymbol{k} \times \tilde{\boldsymbol{j}})_{x} \tilde{\psi}_{0}+k_{x} k_{z}(\boldsymbol{k} \times \tilde{\boldsymbol{j}})_{z} \tilde{\psi}_{2}^{m}\right), \\
\tilde{H}_{y}=-i\left((\boldsymbol{k} \times \tilde{\boldsymbol{j}})_{y} \tilde{\psi}_{0}+k_{y} k_{z}(\boldsymbol{k} \times \tilde{\boldsymbol{j}})_{z} \tilde{\psi}_{2}^{m}\right), \\
\tilde{H}_{z}=-i(\boldsymbol{k} \times \tilde{\boldsymbol{j}})_{z} \tilde{\psi}_{1}^{m},
\end{array}\right.
\end{aligned}
$$




$$
\left((\boldsymbol{k} \times \tilde{j})_{z} \equiv k_{x} \tilde{j}_{y}-k_{y} \tilde{j}_{x}\right) .
$$

It is possible to represent the electromagnetic fields in (75) and (76) in vector form as following

$$
\begin{gathered}
\tilde{\boldsymbol{E}}=\left(i \varepsilon \varepsilon_{0} \omega\right)^{-1}\left(k_{0}^{2}\left(\tilde{\boldsymbol{j}} \tilde{\psi}_{0}+\boldsymbol{k} \times \mathbf{e}_{z}\left(\boldsymbol{k} \times \tilde{\boldsymbol{j}}_{\perp}\right)_{z} \tilde{\psi}_{2}^{m}\right)-\boldsymbol{k}(\boldsymbol{k} \tilde{\boldsymbol{j}}) \tilde{\psi}_{0}\right), \\
\tilde{\boldsymbol{H}}=i\left(\mathbf{e}_{z} k_{z}-\boldsymbol{k}\right) k_{z}\left(\boldsymbol{k} \times \tilde{\boldsymbol{j}}_{\perp}\right)_{z} \tilde{\psi}_{2}^{m}+i \mathbf{e}_{z}\left(\boldsymbol{k} \times \tilde{\boldsymbol{j}}_{\perp}\right)_{z}\left(\tilde{\psi}_{0}-\tilde{\psi}_{1}^{m}\right)-i \boldsymbol{k} \times \tilde{\boldsymbol{j}} \tilde{\psi}_{0},
\end{gathered}
$$

where

$$
\tilde{j}=\tilde{j}_{\perp}+\tilde{j}_{0}, \quad \tilde{j}_{0}=\left(0,0, \tilde{j}_{z}\right), \quad \tilde{j}_{\perp}=\left(\tilde{j}_{x}, \tilde{j}_{y}, 0\right), \quad k_{0}^{2}=\omega^{2} \varepsilon_{0} \varepsilon \mu \mu_{0}, \quad k_{n}^{2}=k_{0}^{2} \frac{\mu_{1}}{\mu} .
$$

It should be noted that the following useful formulae follow from (12), (73) and (74):

$$
\begin{gathered}
\tilde{\psi}_{0}-\tilde{\psi}_{1}^{m}=\left(k_{0}^{2}-k_{z}^{2}\right) \tilde{\psi}_{2}^{m}, \\
\tilde{\psi}_{0}-\frac{\mu_{1}}{\mu} \tilde{\psi}_{1}^{m}=\left(k_{x}^{2}+k_{y}^{2}\right) \tilde{\psi}_{2}^{m} .
\end{gathered}
$$

With the help of identity in (79) and (80), the last equation (78) can be represented as

$$
\tilde{\boldsymbol{H}}=i\left(k_{0}^{2} \mathbf{e}_{z}-k_{z} \boldsymbol{k}\right)\left(\boldsymbol{k} \times \tilde{\boldsymbol{j}}_{\perp}\right) z \tilde{\psi}_{2}^{m}-i(\boldsymbol{k} \times \tilde{\boldsymbol{j}}) \tilde{\psi}_{0} .
$$

Using the property of convolution (17) and considering inverse Fourier transformation it is possible to get the solution of the Maxwell equations in form (18).

So, after the inverse Fourier transformations from (77) and (81) we obtain:

$$
\begin{gathered}
\boldsymbol{E}=\left(i \varepsilon \varepsilon_{0} \omega\right)^{-1}\left(\left(k_{0}^{2}+\nabla \nabla\right) \boldsymbol{j} * \psi_{0}-k_{0}^{2} \nabla \times\left(\mathbf{e}_{z}\left(\nabla \times \boldsymbol{j}_{\perp}\right) z\right) * \psi_{2}^{m}\right), \\
\boldsymbol{H}=\left(k_{0}^{2} \mathbf{e}_{z}+\frac{\partial}{\partial z} \nabla\right)\left(\nabla \times \boldsymbol{j}_{\perp}\right) z * \psi_{2}^{m}-\nabla \times \boldsymbol{j} * \psi_{0} .
\end{gathered}
$$

This solution can be written in the form of the sum of two solutions:

$$
E=E_{1}+E_{2}, \quad H=H_{1}+H_{2} .
$$

It should be noted that the first of them is the 'isotropic' solution. It is defined by Green's function $\psi_{0}$ and the density of the current $j_{0}$ directed parallel to axis $z$ (of the anisotropy):

$$
\left\{\begin{array}{l}
\boldsymbol{E}_{1}=\left(i \varepsilon \varepsilon_{0} \omega\right)^{-1}\left(\nabla \nabla+k_{0}^{2}\right)\left(\psi_{0} * \boldsymbol{j}_{0}\right), \\
\boldsymbol{H}_{1}=-\nabla \times\left(\psi_{0} * \boldsymbol{j}_{0}\right),
\end{array}\right.
$$

where $\psi_{0}$ is the Green's function (22). The second solution can be written by using the component of the density of the current $j_{\perp}$ perpendicular to axis $z$ and the Green's functions $\psi_{0}$ and $\psi_{2}^{m}$ :

$$
\left\{\begin{array}{l}
\boldsymbol{E}_{2}=-\frac{i}{\varepsilon \varepsilon_{0} \omega}\left(\left(k_{0}^{2}+\nabla \nabla\right) \boldsymbol{j}_{\perp} * \psi_{0}-k_{0}^{2} \nabla \times\left(\mathbf{e}_{z}\left(\nabla \times \boldsymbol{j}_{\perp}\right) z\right) * \psi_{2}^{m}\right), \\
\boldsymbol{H}_{2}=\left(k_{0}^{2} \mathbf{e}_{z}+\frac{\partial}{\partial z} \nabla\right)\left(\nabla \times \boldsymbol{j}_{\perp}\right) z * \psi_{2}^{m}-\nabla \times \boldsymbol{j}_{\perp} * \psi_{0},
\end{array}\right.
$$




$$
\begin{gathered}
\psi_{1}^{m}=\mathrm{F}^{-1}\left[\tilde{\psi}_{1}^{m}\right]=-\frac{1}{4 \pi} \sqrt{\frac{\mu}{\mu_{1}}} \frac{\exp \left(i k_{n} r^{\prime}\right)}{r^{\prime}}, \\
\psi_{2}^{m}=\left(\frac{\mu}{\mu_{1}}-1\right) \psi_{0} * \psi_{1}^{m}, \\
r^{\prime}=\sqrt{x^{2}+y^{2}+\frac{\mu}{\mu_{1}} z^{2}} .
\end{gathered}
$$

Furthermore, the following transformation is valid similarly to (45):

$$
\mathrm{F}^{-1}\left[\tilde{\psi}_{2}^{m}\right]=\mathrm{F}^{-1}\left[\tilde{\psi}_{0}\left(k_{0}^{2}-k_{z}^{2}\right)^{-1}\right]-\mathrm{F}^{-1}\left[\tilde{\psi}_{1}^{m}\left(k_{0}^{2}-k_{z}^{2}\right)^{-1}\right] .
$$

We find that function $\psi_{2}^{m}$, Eqn. (87) is given by:

$$
\begin{aligned}
\psi_{2}^{m}=- & \frac{i}{8 \pi k_{0}}\left(e^{i k_{0} z}\left(\operatorname{Ci}\left(k_{0}(r-z)\right)+i \operatorname{si}\left(k_{0}(r-z)\right)\right)+e^{-i k_{0} z}\left(\operatorname{Ci}\left(k_{0}(r+z)\right)+i \operatorname{si}\left(k_{0}(r+z)\right)\right)-\right. \\
& \left.-e^{i k_{0} z}\left(\operatorname{Ci}\left(k_{n} r^{\prime}-k_{0} z\right)+i \operatorname{si}\left(k_{n} r^{\prime}-k_{0} z\right)\right)-e^{-i k_{0} z}\left(\operatorname{Ci}\left(k_{n} r^{\prime}+k_{0} z\right)+i \operatorname{si}\left(k_{n} r^{\prime}+k_{0} z\right)\right)\right) .
\end{aligned}
$$

It should be noted that $\psi_{2}$ and $\psi_{2}^{m}$ are similar for magnetic and dielectric crystals.

\subsection{Radiation patterns of Hertzian radiator in magnetically anisotropic media}

On the basis of the results obtained above, we consider now numerical results for the radiation of the electric dipole.

The dipole moment of point electric dipole is given by $p$, Eqn. (52). It corresponds to the current density defined by means of the Dirac delta-function (53).

The expression of the electromagnetic field for electric radiator will take the following form as for isotropic medium, when the direction of the dipole moment is parallel to the axis $z$ $\left(p=p_{0}\right)($ Fig. 7$)$ :

$$
\left\{\begin{array}{l}
\boldsymbol{E}_{1}=-\left(i \varepsilon \varepsilon_{0}\right)^{-1}\left(\nabla \nabla+k_{0}^{2}\right)\left(\psi_{0} \boldsymbol{p}_{0}\right), \\
\boldsymbol{H}_{1}=i \omega \nabla \times\left(\psi_{0} \boldsymbol{p}_{0}\right) .
\end{array}\right.
$$

Also when the direction of the dipole moment is perpendicular to axis $z$, we obtain $\left(\boldsymbol{p}=\boldsymbol{p}_{\perp}\right)$ (Fig. 8, Fig. 9):

$$
\left\{\begin{array}{l}
\boldsymbol{E}_{2}=\left(\varepsilon \varepsilon_{0}\right)^{-1}\left(k_{0}^{2} \nabla \times\left(\mathbf{e}_{z}\left(\nabla \times \boldsymbol{p}_{\perp} \psi_{2}^{m}\right)_{z}\right)-\left(k_{0}^{2}+\nabla \nabla\right)\left(\boldsymbol{p}_{\perp} \psi_{0}\right)\right), \\
\boldsymbol{H}_{2}=\left(k_{0}^{2} \mathbf{e}_{z}+\frac{\partial}{\partial z} \nabla\right)\left(\nabla \times \boldsymbol{p}_{\perp} \psi_{2}^{m}\right)_{z}-\nabla \times\left(\boldsymbol{p}_{\perp} \psi_{0}\right) .
\end{array}\right.
$$

\subsection{Radiation patterns of point magnetic dipole in magnetically anisotropic media}

On the basis of the obtained results, we consider now radiation of point magnetic dipole moment. For a point radiator with the oscillating magnetic dipole moment, similarly to the magnetic dipole case in one-axis crystals, Eqn. (60), the electric current density is defined by using Dirac's delta-function, Eqn. (61). It is possible to express the magnetic dipole moment $m$ in the form of the sum of two components of magnetic moment:

$$
m=m_{0}+m_{\perp}, \quad m_{0}=\mathbf{e}_{z} m_{z} .
$$



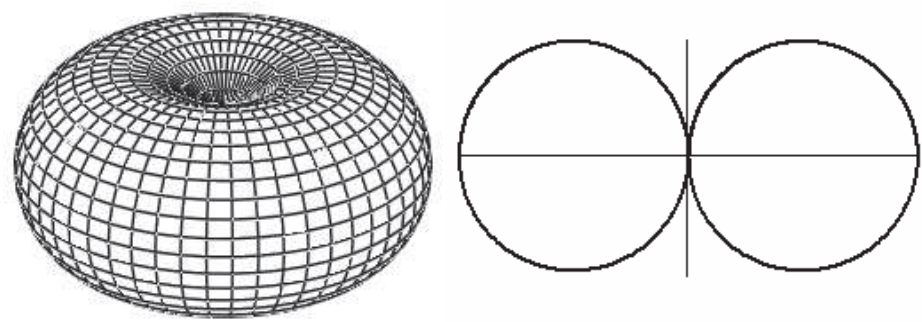

Fig. 7. Directivity diagram. Electric dipole moment is parallel to the axis $z\left(p=p_{0}\right)$

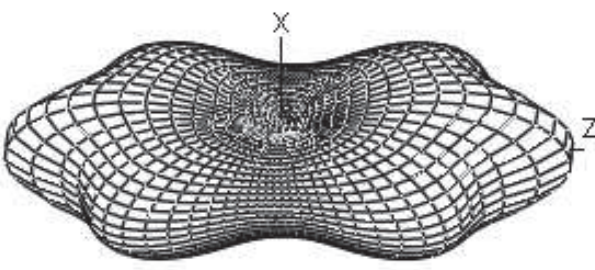

(a) Case $r=3$

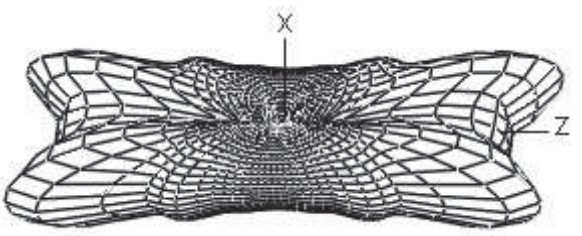

(c) Case $r=9$

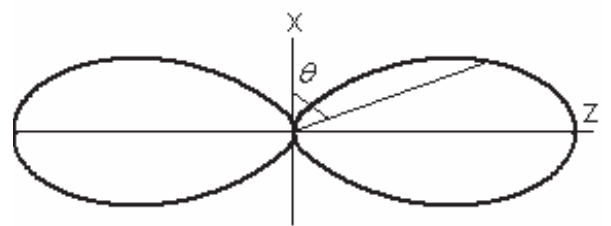

(b) View in meridian surface $r=3, \varphi=\pi / 2$

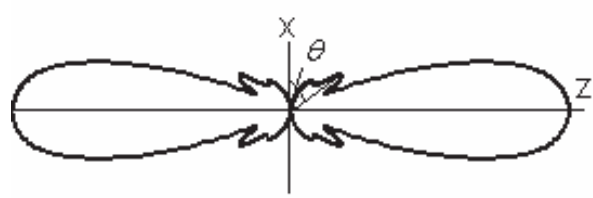

(d) View in meridian surface $r=9, \varphi=\pi / 2$

Fig. 8. Directivity diagram. The axis of electric dipole is perpendicular to axis $z\left(p=p_{\perp}\right)$, $\mu_{1} / \mu=9$

Components of a current density, Eqn. (61), have the following form:

$$
j_{0}=\mathbf{e}_{z}\left(m_{y} \frac{\partial}{\partial x}-m_{x} \frac{\partial}{\partial y}\right) \delta(\boldsymbol{r})
$$

and

$$
j_{\perp}=\left(\mathbf{e}_{x}\left(m_{z} \frac{\partial}{\partial y}-m_{y} \frac{\partial}{\partial z}\right)+\mathbf{e}_{y}\left(m_{x} \frac{\partial}{\partial z}-m_{z} \frac{\partial}{\partial x}\right)\right) \delta(\boldsymbol{r}) .
$$

Case $m=m_{0}$.

Relation between density of an electric current $j_{\perp}$ and the magnetic dipole moment is defined from (93) in case when the magnetic dipole moment $m$ is directed lengthwise $z$ :

$$
j_{\perp}=m_{z}\left(\mathbf{e}_{x} \frac{\partial}{\partial y}-\mathbf{e}_{y} \frac{\partial}{\partial x}\right) \delta(\boldsymbol{r}) .
$$






(a) Case $r=1$

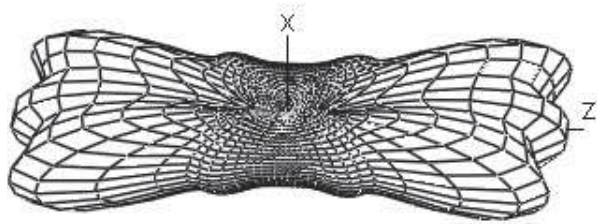

(c) Case $r=5$



(b) View in meridian surface $r=1, \varphi=\pi$

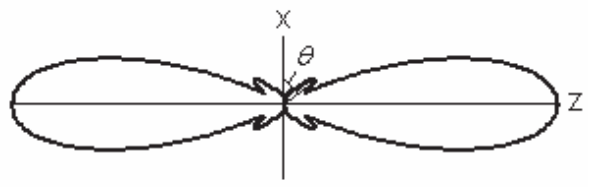

(d) View in meridian surface $r=5, \varphi=\pi$

Fig. 9. Directivity diagram. The axis of electric dipole is perpendicular to axis $z\left(p=p_{\perp}\right)$, $\mu_{1} / \mu=15$

It should be noted that the following useful formula hold:

$$
\nabla \boldsymbol{j}_{\perp}=0, \quad j_{0}=0 .
$$

Taking into account (96), intensities of the electromagnetic field by the magnetic dipole moment are defined from the solutions (85) in this case, as following:

$$
\left\{\begin{array}{l}
\boldsymbol{E}=-i \mu_{0} \mu_{1} \omega m_{0} \nabla \times\left(\mathbf{e}_{z} \psi_{1}^{m}\right), \\
\boldsymbol{H}=m_{0} \Delta \psi_{1}^{m}+m_{0}\left(\frac{\mu_{1}}{\mu}-1\right) \frac{\partial^{2}}{\partial z^{2}} \psi_{1}^{m}-m_{0} \frac{\mu_{1}}{\mu} \frac{\partial}{\partial z} \nabla \psi_{1}^{m} .
\end{array}\right.
$$

We have taken advantage of the next formulae which followed from (79) after inverse Fourier transformation

$$
\begin{gathered}
\frac{\mu_{1}}{\mu} \psi_{1}^{m}=\psi_{0}+\left(\frac{\partial^{2}}{\partial x^{2}}+\frac{\partial^{2}}{\partial y^{2}}\right) \psi_{2}^{m}, \\
\psi_{0}=\psi_{1}^{m}+\left(\frac{\partial^{2}}{\partial z^{2}}+k_{0}^{2}\right) \psi_{2}^{m} .
\end{gathered}
$$

Directional diagrams are represented in Fig. 10.

\section{Case $m=m_{\perp}$.}

For the point magnetic dipole moment $m_{\perp}$ which is perpendicular to axis $z$, by substituting (61) ( or (93) and (94)) in (84) and (85), we define intensities of electromagnetic field as following (Fig. 11, Fig. 12):

$$
\left\{\begin{array}{l}
\boldsymbol{E}=i \mu_{0} \mu \omega\left(\frac{\partial}{\partial z}\left\{\boldsymbol{m}_{\perp} \times \mathbf{e}_{z} \frac{\mu_{1}}{\mu} \psi_{1}^{m}-\nabla_{\perp}\left(\nabla \times \psi_{2}^{m} \boldsymbol{m}_{\perp}\right)_{z}\right\}-\mathbf{e}_{z}\left(\nabla \times \psi_{0} \boldsymbol{m}_{\perp}\right) z\right), \\
\boldsymbol{H}=\left(k_{0}^{2} \mathbf{e}_{z}+\frac{\partial}{\partial z} \nabla\right) \frac{\partial}{\partial z} \nabla\left(\boldsymbol{m}_{\perp} \psi_{2}^{m}\right)-\nabla \times \nabla \times\left(\boldsymbol{m}_{\perp} \psi_{0}\right) .
\end{array}\right.
$$




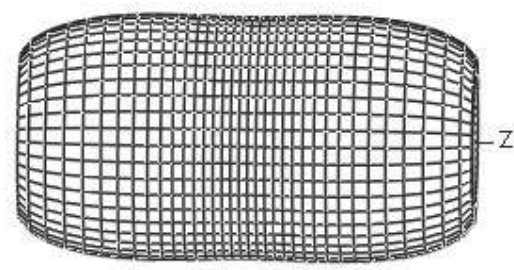

(a) Case $\mu_{1} / \mu=7$

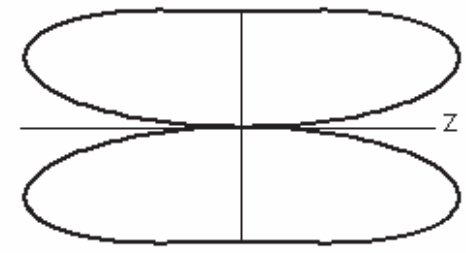

(b) View in meridian surface $(\varphi=0)$

Fig. 10. Directivity diagram. The axis of magnetic dipole is parallel to axis $z\left(m=m_{0}\right)$

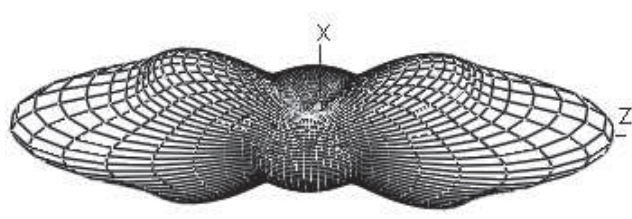

(a) Case $r=5$



(c) Case $r=10$

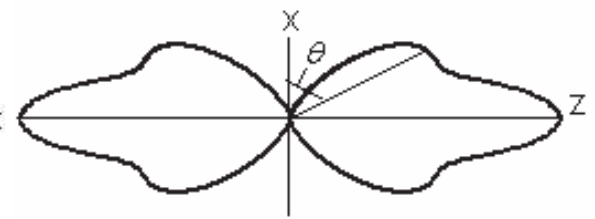

(b) View in meridian surface $r=5, \varphi=\pi / 2$

Fig. 11. Directivity diagram. The axis of magnetic dipole is perpendicular to $z, \mu_{1} / \mu=7$

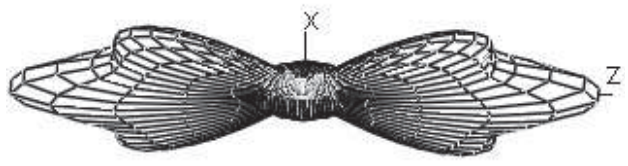

(a) Case $r=5$

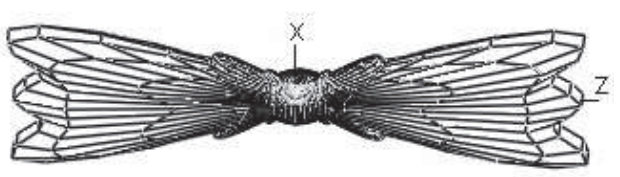

(c) Case $r=10$

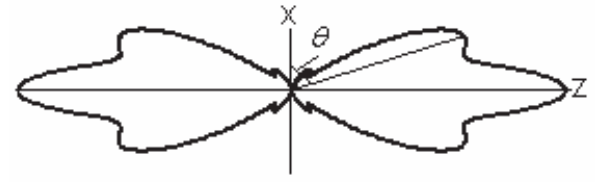

(b) View in meridian surface $r=6, \varphi=\pi / 2$

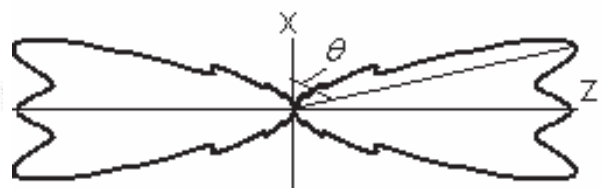

(d) View in meridian surface $r=10, \varphi=\pi / 2$

Fig. 12. Directivity diagram. The axis of magnetic dipole is perpendicular to $z, \mu_{1} / \mu=15$

The numerical calculation of the solution of Maxwell equations satisfies the energy conservation law. Numerical computation shows that time average value energy flux on a 
surface of sphere from a point dipole remains independent from its radius, with very high accuracy. As shown in the electric dipole directional diagrams, medium becomes isotropic for such radiator if its moment is directed along anisotropy axis.

The dipole pattern in isotropic media is shown in Fig. 1 and directional diagram itself possesses the rotation symmetry. However the point magnetic moment does not possess such property.

When $\mu_{1}$ approaches $\mu$, the potential $\psi_{2}$ tends to zero and the well-known expressions of electromagnetic field in form (57), (58) and (59) follow from (84) and (85).

The obtained generalized solutions of the Maxwell equations are valid for any values of $\mu_{1}$ and $\mu$, also near the sources of the electromagnetic waves, described by discontinuous and singular functions.

\section{Conclusion}

We were able to solve Maxwell's equations for uniaxial anisotropic medium actually showing that the exact general solution in vector form is given by integral convolutions of the fundamental solutions $\psi_{0}, \psi_{1}$ and $\psi_{2}$ with external arbitrary current density, without using the scaling procedure to a corresponding vacuum field and the dyadic Green's functions. In the particular case of a current density, the radiation field of a dipole is considered in greater detail. That method may be useful in analytical treatment of corresponding boundary value problems.

\section{Acknowledgment}

I wish to thank Professor Panayiotis Frangos, School of Electrical and Computer Engineering, National Technical University of Athens, Greece, for providing me with the encouragement to pursue research in this field.

\section{References}

Alekseyeva, L. A. \& Sautbekov, S. S. (1999). Fundamental Solutions of Maxwell's Equations. Diff. Uravnenia,Vol. 35, No. 1, 125-127.

Alekseyeva, L. A. \& Sautbekov, S. S. (2000). Method of Generalized Functions For Solving Stationary Boundary Value Proplems For Maxwell's Equations. Comput. Math. and Math. Phys., Vol. 40, No. 4, 582-593.

Barkeshli, S. (1993). An efficient asymptotic closed-form dyadic Green's function for grounded double-layered anisotropic uniaxial material slabs, J. Electromagnetic Waves and Applications, Vol. 7, No. 6, 833-856.

Born, M. \& Wolf, E. (1999). Principles of Optics. Electromagnetic Theory of Propagation, Interference and Dffraction of Light, 7th ed. Cambridge U. Press, Cambridge.

Bunkin, F.V. (1957). On Radiation in Anisotropic Media. Sov. Phys. JETP Vol. 5, No.2, 277-283.

Chen, H. C. (1983). Theory of Electromagnetic Waves, McGraw-Hill, New York.

Clemmow, P.C. (Jun. 1963a). The theory of electromagnetic waves in a simple anisotropic medium, Proc. IEE, pp. 101-106, Vol. 110, No. 1, Jun 1963.

Clemmow, P.C. (Jun. 1963b). The resolution of a dipole field in transverse electric and transverse magnetic waves, Proc. IEE, pp. 107-111, Vol. 110, No. 1, Jun 1963. 
Cottis, P. G. \& Kondylis, G. D. (Feb. 1995). Properties of the dyadic Green's function for an unbounded anisotropic medium, IEEE Trans. Ant. Prop., Vol. 43, No. 2, Feb. 1995, 154-161.

Kogelnik, H. \& Motz, H. (1963). Electromagnetic Radiation from Sources Embedded in an Infinite Anisotropic Medium and the Significance of the Poynting Vector, Proc. Symp. on Electromagnetic Theory and Antennas, pp. 477-493, 1963, Pergamon Press, New York.

Kong, J. A. (1986). Electromagnetic Wave Theory, The 2nd ed., John Wiley and Sons, New York.

Lee, J. K. \& Kong, J. A. (1983). Dyadic Green's functions for layered anisotropic medium. Electromagnetics, Vol. 3, 111-130.

Ren, W. (Jan. 1993). Contributions to the Electromagnetic Wave Theory of Bounded Homogeneous Anisotropic Media. Physical Review E, Vol. 47, No. 1, 664-673.

Sautbekov, S.; Kanymgazieva, I. \& Frangos, P. (2008). The generalized solutions of Maxwell equations for the uniaxial crystal, Journal of Applied Electromagnetism (JAE), Vol. 10, No. 2, 43-55, ISSN 1392-1215.

Uzunoglu, N. K.; Cottis, P. G. \& Fikioris, J. G. (Jan. 1985). Excitation of Electromagnetic Waves in a Gyroelectric Cylinder, IEEE Trans. Ant. Propa., Vol. AP-33, No. 1, 90-99.

Vladimirov, V. S. (2002). Methods of the theory of generalized functions, Taylor and Francis, ISBN 0-415-27356-0, London.

Weiglhofer, W. S. (Feb. 1990). Dyadic Greens functions for general uniaxial media, IEE Proc. H., Vol. 137, No. 1, 5-10.

Weiglhofer, W. S. (1993). A dyadic Green's function representation in electrically gyrotropic media, $A E \ddot{U}$, Vol. 47, No. 3, 125-130. 


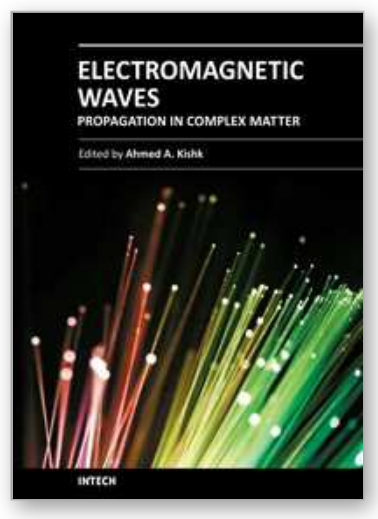

\author{
Electromagnetic Waves Propagation in Complex Matter \\ Edited by Prof. Ahmed Kishk
}

ISBN 978-953-307-445-0

Hard cover, 292 pages

Publisher InTech

Published online 24, June, 2011

Published in print edition June, 2011

This volume is based on the contributions of several authors in electromagnetic waves propagations. Several issues are considered. The contents of most of the chapters are highlighting non classic presentation of wave propagation and interaction with matters. This volume bridges the gap between physics and engineering in these issues. Each chapter keeps the author notation that the reader should be aware of as he reads from chapter to the other.

\title{
How to reference
}

In order to correctly reference this scholarly work, feel free to copy and paste the following:

Seil Sautbekov (2011). The Generalized Solutions of a System of Maxwell's Equations for the Uniaxial Anisotropic Media, Electromagnetic Waves Propagation in Complex Matter, Prof. Ahmed Kishk (Ed.), ISBN: 978-953-307-445-0, InTech, Available from: http://www.intechopen.com/books/electromagnetic-wavespropagation-in-complex-matter/the-generalized-solutions-of-a-system-of-maxwell-s-equations-for-the-uniaxialanisotropic-media

\section{INTECH}

open science | open minds

\section{InTech Europe}

University Campus STeP Ri

Slavka Krautzeka 83/A

51000 Rijeka, Croatia

Phone: +385 (51) 770447

Fax: +385 (51) 686166

www.intechopen.com

\section{InTech China}

Unit 405, Office Block, Hotel Equatorial Shanghai

No.65, Yan An Road (West), Shanghai, 200040, China

中国上海市延安西路65号上海国际贵都大饭店办公楼 405 单元

Phone: +86-21-62489820

Fax: +86-21-62489821 
(C) 2011 The Author(s). Licensee IntechOpen. This chapter is distributed under the terms of the Creative Commons Attribution-NonCommercialShareAlike-3.0 License, which permits use, distribution and reproduction for non-commercial purposes, provided the original is properly cited and derivative works building on this content are distributed under the same license. 